\title{
Visual Discrimination Learning in the Jumping Spider Phidippus regius
}

\author{
Massimo De Agrò ${ }^{1 *}$, Lucia Regolin ${ }^{1}$, and Enzo Moretto ${ }^{2,3}$ \\ ${ }^{1}$ Department of General Psychology, University of Padua, Padua, Italy \\ 2 Esapolis' Living Insects Museum of the Padua Province, Padua, Italy \\ ${ }^{3}$ Butterfly Arc Ltd., Padua, Italy \\ *Corresponding author (Email: massimo.deagro@phd.unipd.it)
}

Citation - De Agrò, M., Regolin, L., \& Moretto, E. (2017). Visual discrimination learning in the jumping spider Phidippus regius. Animal Behavior and Cognition, 4(4), 413-424. https://dx.doi.org/10.26451/abc.04.04.02.2017

\begin{abstract}
Over the past decade, research in comparative psychology has increasingly focused on non-vertebrate models of cognition. Jumping spiders provide excellent models for the study of visually mediated behaviors, such as associative learning or the navigation of complex environments. Here, we tested visual and memory abilities of Phidippus regius to discriminate between artificial geometrical stimuli and to generalize the learned discrimination to illusory stimuli, using the amodal completion mechanism. Spiders were first trained to associate one shape (' $\mathrm{X}$ ' or 'O') with a reward (sugar water), whilst the other shape was associated with an aversive taste (acidic water). Spiders were then asked to choose between the two shapes in the absence of any reward or punishment. They were then presented with an occluded version of the previously rewarded shape to test for the presence of amodal completion. Spiders were able to learn the discrimination task, although the association was not transferred to the illusory stimulus. This study provides the first demonstration of shape discrimination learning in a jumping spider. The results of the test on the illusory shape are discussed considering that either the spiders' visual system may not require amodal completion or they could have the tendency to learn the shape associated with the aversive taste rather than that associated with the reward.
\end{abstract}

Keywords - Jumping Spiders, Salticidae, Phidippus regius, Visual illusion, Amodal completion, Visual discrimination learning

During the past two decades, the literature on the cognitive abilities of arthropods has significantly grown. Arthropods appear able to perform a variety of complex tasks, ranging from spatial navigation (Menzel et al., 2005; Wehner, 2003) to object perception (van Hateren, Srinivasan, \& Wait, 1990), learning (Hammer \& Menzel, 1995), numerical competences (Cross \& Jackson, 2017; Dacke \& Srinivasan, 2008; Nelson \& Jackson, 2012) and even more complex skills, such as self-recognition (Cammaerts \& Cammaerts, 2015) and the cultural spreading of learned abilities (Alem et al., 2016). Among arthropods, a family of spiders has caught the interest of scientists: Salticidae. They display interesting behaviors that likely depend on highly flexible and complex cognitive abilities (Herberstein, 2011). For example, spiders of the genus Portia seem able to retain in their memory the position of prey in the environment (Tarsitano, 2006; Tarsitano \& Andrew, 1999; Tarsitano \& Jackson, 1997) and even modify their behavior when facing unexpected scenarios, as if they held expectations about certain events (Cross \& Jackson, 2014).

Jumping spiders rely primarily on vision to hunt and navigate the environment. They, in fact, possess a highly developed visual system, which consists of eight camera eyes. The two principal eyes (anterior medial eyes, AME) have the highest spatial resolution of all, even though they have a narrow 
visual field. They both consist of a long optic tube, which can be moved by a set of antagonist muscles. This makes the spider able to focus on different portions of the environment without needing to move the body, effectively extending their visual field. Of the other six eyes (called secondary eyes), the anterior lateral eyes (ALE) seem to be the most interesting when talking about object detection: they are forward facing, and their visual fields overlap. They are probably needed to orient the spider toward stimuli (like the other secondary eyes) and to guide the movement of the AME (Harland, Li, \& Jackson, 2012).

Land (1969) first described the movement of the spider and its eyes while inspecting visual stimuli. In particular, the "scanning" behavior seems to play a crucial role in object recognition: This behavior occurs only after an object has been detected and fixated, and it consists of the movement of the retina across the target. Land (1969) stated that "An important task that the retinae are performing during scanning is therefore going to be the detection of lines or contours with particular orientations and in appropriate positions" (p. 490). This suggests that the local features of stimuli are crucial for object recognition, in particular, in the task of categorizing prey. Dolev and Nelson (2014) tested Evarcha culicivora to assess which elements of a figure are necessary for the spider to trigger a stalking reaction. The authors identified some local features crucial for responding to a mosquito (preferred prey of Evarcha). In a more recent study, Dolev and Nelson (2016) further analyzed the attention of Evarcha to those local features, testing the preference of the animals between simple stimuli containing features of preferred prey (mosquito) and a realistic representation of other, non-preferred prey (flies). Evarcha showed a clear preference for the first class of stimuli and chose the simple features independent of their global configuration (but also showing selective attention to the relative orientation of the elements present in each stimulus). On the contrary, Hypoblemum albovittatum, a generalistic predator, in all instances preferred the realistic representations to the simpler stimuli. Dolev and Nelson (2016) suggested that the privileged use of local features could represent an adaptation for a specialist predator to locate more effectively its preferred prey. This would not be the case for a generalistic predator, which could instead benefit from using the general configuration of the image to identify prey. However, in the study by Dolev and Nelson (2016), realistic (and perceptually richer) pictures were confronted with much simpler shapes (made of lines and circles). Stimuli were not paired so as to test for the use of the actual configuration (as would be obtained when globally different but locally identical stimuli are confronted), except for one comparison (conditions A and F of their study). No conclusions could be drawn as both species performed at random (i.e., they did not stalk preferentially any of the two stimuli presented).

The studies mentioned above (Dolev \& Nelson, 2014, 2016; Land, 1969) focused on the cues that the spiders used to distinguish prey from non-prey, and they demonstrated that local features can be crucial for triggering the spider's stalking response. But what about the general mechanisms underlying the perception of stimuli which are not necessarily prey? Would the local features still be crucial, or would the spider in this case disregard the features per se and instead respond to the whole configuration (i.e., to a particular arrangement of the local features)? Many animal species were shown to preferentially respond to the global configuration in object recognition, and often times this was tested by exploiting a mechanism called 'amodal completion.' Through amodal completion, an animal can perceive a figure as a whole even if another object conceals a portion of it (Michotte, Thines, \& Crabbe, 1964). Mammals (Kanizsa, Renzi, Conte, Compostela, \& Guerani, 1993; Sato, Kanazawa, \& Fujita, 1997), birds (Regolin \& Vallortigara, 1995), fish (Sovrano \& Bisazza, 2008) and even invertebrates (van Hateren et al., 1990; Zylinski, Darmaillacq, \& Shashar, 2012) use this mechanism just as humans do (Kanizsa, 1979).

We investigated jumping spiders' ability to acquire the discrimination between two abstract, geometrical shapes through associative learning. We tested Phidippus regius (Koch, 1846), a species belonging to the family of Salticidae that has been successfully tested through a variety of methodologies, such as conditioning (Jakob, Skow, Haberman, \& Plourde, 2007; Liedtke \& Schneider, 2014; Peckmezian \& Taylor, 2015a), using simulated environments (Peckmezian \& Taylor, 2015b) and even using neurophysiological recording techniques (Menda, Shamble, Nitzany, Golden, \& Hoy, 2014). A previous study (Bednarski, Taylor, \& Jakob, 2012) tested the ability of jumping spiders to discriminate between the moving image of a cricket (meaningful) and a moving rectangle (abstract), but the animals seemed unable to learn to discriminate between the two. However, the focus of the experiment was the detection 
of the motion of stimuli, rather than their shape. In our study, once the spiders had undergone the training phase, we assessed their free choice in unrewarded test trials to assess discrimination learning. If Phidippus regius was able to learn to associate a neutral visual stimulus (an abstract shape) with a reward, its performance was expected to be higher than the chance level (i.e., the spider was expected to approach the previously positively rewarded shape and disregard the previously negatively rewarded shape). Successively, we tested spiders' ability to generalize the learned response to a partly concealed version of the stimulus reinforced during training. If this species could perform amodal completion similarly to humans (and also to many other species) when confronted with an occluded version of the previously positively rewarded shape, the spiders were expected to choose to approach such a stimulus and not its 'broken,' non-occluded version. Both stimuli in this comparison would present identical local features, but only the occluded stimulus would trigger shape completion by the human eye. If, on the other hand, the spider's vision relied on local features, we expected a random choice between the two stimuli presented in this comparison.

\section{Methods}

\section{Subjects}

Eighteen adult female spiders (instar 8 -9) were used for the experiment. Five of them came from one eggsac and thirteen from another one. The eggsacs were laid by two different spiders. The subjects were housed individually in test tubes upon emerging from the mothers' nest. Then, after the first molt, the spiders were individually placed in plastic boxes (each with dimensions of $7 \times 16 \times 6 \mathrm{~cm}$ ), with each featuring a portion of a cardboard egg container to provide shelter and a walking surface, and a wet sponge to provide humidity and drinking water. The dimensions and contents of boxes were chosen according to the findings of Carducci and Jakob (2000) given the fact that jumping spiders show better performance when raised in larger, enriched environments. The box walls were transparent and with holes on the sides to allow airflow. Light was shed on the boxes via neon lamps with natural light (5000 kelvin color temperature, 36 watts, 3350 lumens). The light cycle was set at $12 \mathrm{hr}$ light and $12 \mathrm{hr}$ dark. We fed one to three Drosophila melanogaster every three days between the second and third instars. After this, the spiders were fed Tenebrio molitor larvae (one each week) that were similar in size to the spiders. The larvae were bred in the laboratory and fed a specific diet to ensure their best development. Prior to the experiment, the spiders had no interactions among one another (apart from the days spent inside the nests of the mothers). Moreover, they had no previous experience with the types of stimuli presented during the experiment, either unconditioned and conditioned (e.g., drop and shape as described below).

\section{Apparatus}

The experimental apparatus consisted of a box of the same size as the housing ones. The walls of the box were covered with white paper to insulate the animal from external cues (Figure 1). The lid of the box remained transparent to allow lighting and allow for recording. The spiders were presented with two red-colored drops. One of the drops contained sugar (20\% weight on volume solution), and the other contained citric acid ( $25 \%$ weight on volume solution). The drops were visually identical to the human eye. However, as sugar absorbs ultra-violet light, the two drops could appear different to the spiders (De Voe, 1975). We chose to use these unconditioned stimuli based on the experiment by Liedtke and Schneider (2014), in which they appeared to be good rewards for training jumping spiders. Each drop (approximately $40 \mu \mathrm{l}$ ) was placed on a white plastic square (Figure 2). A vertical piece of plastic was placed on one of the four sides to hide the drop from the spider's visual perspective. On both sides of the vertical wall, on the center, a black shape was glued, either an ' $X$ ' or a ' $O$ '. Both shapes were matched for total area $\left(7 \mathrm{~mm}^{2}\right)$, made via a cutting plotter (Signpal PUMA II) on adhesive plastic (approximately 0.1 mm thick). 


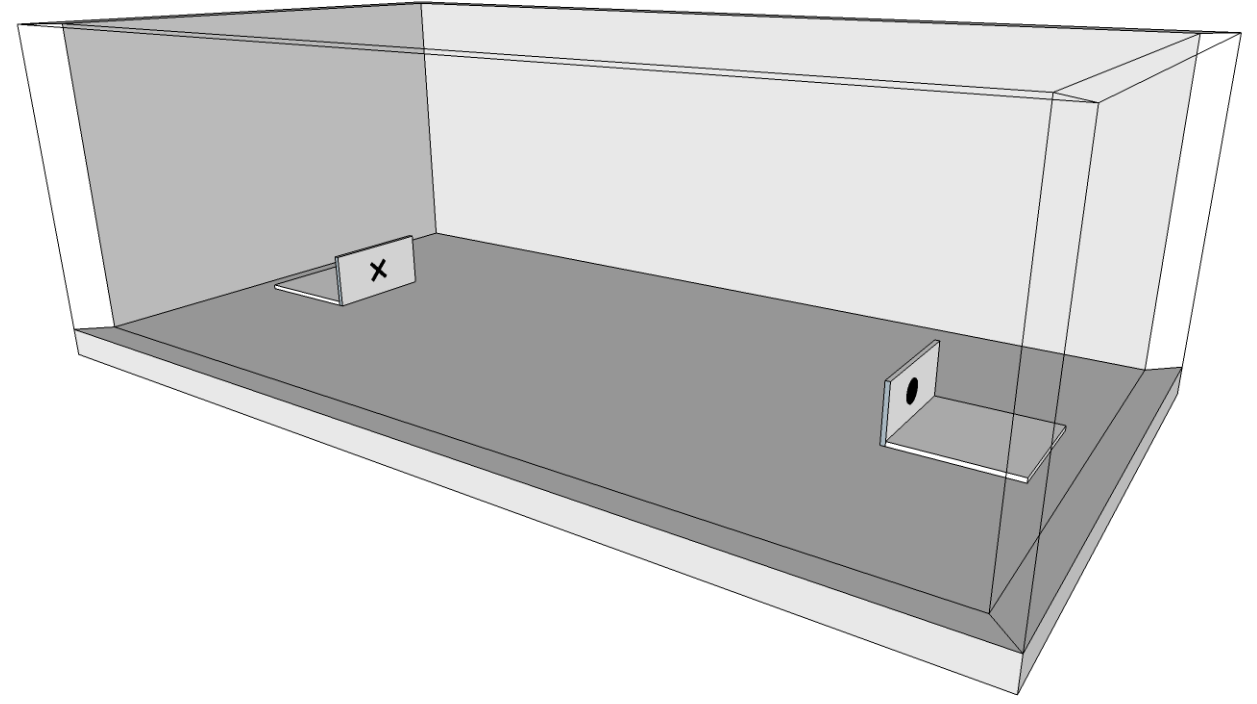

Figure 1. Experimental apparatus with the two platforms ("X" on the left, "O" on the right). The spider's starting position was located in between and exactly at the same distance from either of the two platforms.

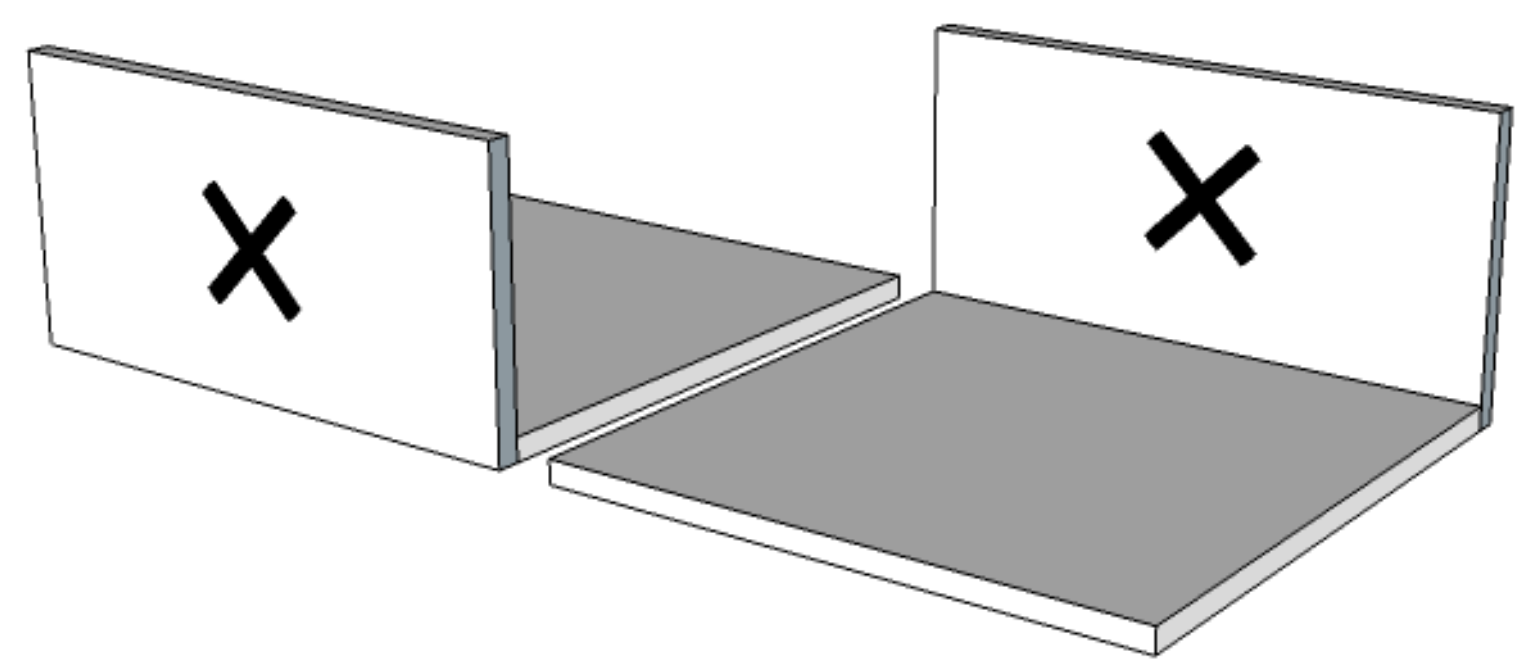

Figure 2. "X" platform, front and back views. The drop is placed in the centre of the base. The base is $20 \mathrm{~mm} \times 20 \mathrm{~mm}, 0.8 \mathrm{~mm}$ thick. The vertical wall is $10 \mathrm{~mm} \times 20 \mathrm{~mm}, 0.8 \mathrm{~mm}$ thick.

\section{Design and Procedure}

Prior to the testing period, the spiders underwent seven days of fasting to ensure a high level of motivation. The day before the testing period, the subjects were placed in an empty testing chamber, in which they remained until the end of the experiment. Each spider underwent a training phase and a testing phase. 
Training Phase. The training phase lasted for seven days. Each day, three trials were performed for each spider, except for the fourth day, during which no test was performed, to allow the spider to regain motivation for the reward. In total, each spider underwent 18 training trials. For each trial, the subject was positioned in the center of the box and covered with a small opaque screen. Then, the two platforms with the two drops, one with citric acid and one with sugar, were placed at the two ends of the box. Finally, the spider was released. Each trial lasted for approximately $45 \mathrm{~min}$, during which the animal was free to move, look at and taste both drops before the trial ended. The timing of the trials was chosen because in pilot trials, our spiders showed a long latency prior to choosing a stimulus. Between trials, a period of approximately 60 min elapsed to avoid excessive stress on the animals. The training shape was randomly assigned to each spider so that half of the subjects were trained on one and the other half on the other shape. To prevent the spider from using external cues to locate the drop of sugar water (i.e., the position of the light) the box was rotated after each trial. Moreover, the position (left or right of the box) of the correct stimulus was changed randomly from trial to trial. Between each trial, the plastic squares were thoroughly cleaned with alcohol to remove any possible trace of sugar or citric acid. Moreover, the stimuli were placed in the box using new latex gloves every time to exclude any possible chemical cues (other than the drop itself).

Testing Phase. After the training phase, a two-day pause took place, during which a damp sponge and small Tenebrio molitor larvae were placed in the box. This was needed, as the sugar water, even though it represented a favorite food of the animals, was not nutrient rich enough. The testing phase lasted between one and three weeks. The number of sets that a spider underwent depended on their age and their level of stress. Younger spiders were considered able to undergo a higher amount of stress. Moreover, some spiders died of old age after the first or second week, so they performed fewer tests. Lastly, if a spider started to lose strength and decrease its activity level due to the lack of nutrient food, the test was stopped, and started again after a week pause with a re-training. Each spider underwent three trials per day. Every three days, a $24 \mathrm{hr}$ interval was observed. As in the training phase, each spider performed a total of 18 trials.

Two-thirds of the trials were completely identical to the training phase: we still presented to the spiders the two different drops with sugar and citric acid (rewarded trials). Those trials were useful for maintaining the learned association between shape and reward. A third of the trials were unrewarded testing trials, during which only red-colored water (with neither sugar nor citric acid) was presented. Removing the reward and the punishment was needed to control for any cues, such as odor, color and taste. Two different types of testing trials were conducted. In the first type, the shapes presented were the same as those used at training ('unrewarded-shape trials'). They were used to assess the outcome of the training phase: If a spider learned the association between the shape and the reward, it was expected to choose the drop behind the stimulus that had been associated with the sugar water.

In the second type of testing trial ('unrewarded-illusion'), the spiders were presented with novel stimuli: An occluded shape (either the ' $\mathrm{X}$ ' or the ' $\mathrm{O}$ ') and a cut version of the same shape (Figure 3). For each spider the shape used was the one that during its training phase had been associated with the sugar water. The occluder in the occluded stimulus was red in color and of a different luminance to the shape to be clearly distinguishable from the actual shape. The local features (i.e., the visible parts of the shape) were identical in the two stimuli, apart from the position of the occluder, so that to distinguish the two stimuli, the animals had to use a global type of visual processing. If a spider relied on amodal completion, it was expected to choose the occluded version of the shape, which would be perceived as the whole shape behind a red bar, whereas the cut stimulus was expected to be seen as a non-complete shape. If the spiders relied on local visual processing, and thus focused on the separate features to identify the shape, they were expected to choose randomly in this condition given the fact that the two stimuli presented identical features.

For the 18 trials, we used a random procedure to determine which trial was rewarded, unrewarded-shape or unrewarded-illusion, maintaining the ratio of two-thirds rewarded trials and onethird unrewarded trials (half with training shapes, and half with illusory vs. cut stimuli). In addition, in the 
testing phase, the boxes were rotated, and the respective positions of the correct and incorrect drops were randomly determined.
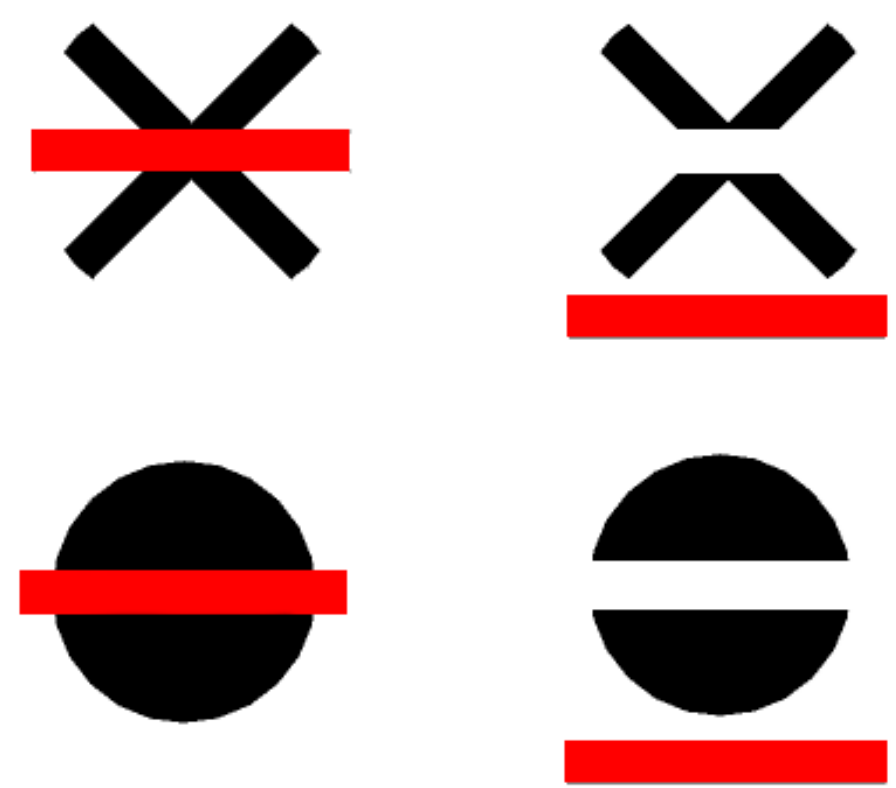

Figure 3. Stimuli presented to the spiders in the "unrewarded-illusion" condition. Spiders trained on the "X" shape were exposed to the 2 stimuli at the top, spiders trained on the "O" shape were exposed to the ones at the bottom. The parts of the shape visible in the stimuli presented together are identical (local features), but stimuli differ in their global configuration if the observer can perform amodal completion. An animal capable of completing occluded objects should in fact perceive as whole only the "occluded" stimulus hence recognizing this as more similar to the shape associated with the reward during training.

We used four cameras to record the behavior of the spiders. Each camera could record four boxes at the same time. For this reason, a maximum of 16 spiders were tested at the same time. From the videos, it was impossible to tell which spider was trained on the ' $\mathrm{X}$ ' and which one on the ' $\mathrm{O}$ ' given the fact that a letter code was used to identify each animal. For this reason, the observer was blind to the experimental conditions. We used the computer program BORIS (Friard \& Gamba, 2016) to code the different behaviors of the spiders during the $45 \mathrm{~min}$ of exposure. We measured the total time, start and end of each event for the following behaviors:

Stasis - The spider does not move at all.

Behind - The spider is positioned behind the wall with the shape, in contact with the plastic platform, with at least one limb.

Drinking - The spider drinks either drop. It is not sufficient to see the spider on top of the drop to code this behavior given the fact that often spiders walk on top of the drop without actually tasting it. When the animal actually drinks, it stops with the chelicera on top of the drop for several seconds and opens the palps wide.

See - We also registered the latency to first detection of each drop. Note that the screen in which the shapes are glued covers the drop, so the spider has to actually move toward the drop to see it. The "drinking" behavior was used as the choice indicator. It was, in fact, a sign of a clear preference of the spider, in addition to being an indicator of motivation (the spider drank only when actually hungry). 


\section{Results}

A total of 828 trials (552 rewarded, 138 unrewarded-shape, 138 unrewarded-illusion) were performed in the testing phase. A total of 769 trials (516 rewarded, 125 unrewarded-shape, 128 unrewarded-illusion) were successfully recorded and analyzed. Unfortunately, due to technical problems, 59 trials were not recorded (most of them from the same day of testing: 3 trials $* 4$ spiders per camera $* 3$ cameras $=36$ ).

Of the trials we analyzed, the spiders drank either drop in 154 trials (93 rewarded, 34 unrewarded-shape, 27 unrewarded-illusion), whereas only in three trials did the subject drink from both drops. The performance on these three trials was discarded from the final analysis because it indicated a lack of choice, just like the performance of the trials in which the spiders did not drink from any drop. The analysis was performed on 151 observations (93 rewarded, 31 unrewarded-shape, 27 unrewardedillusion) (Table 1). Different spiders performed different numbers of trials in the three different conditions of the testing phase. Moreover, many spiders performed only one test each for some conditions (Table 2). Due to the non-independence of the repeated-measures procedure, the individual heterogeneity and the variable individual number of observations, a mixed generalized linear model (GLMM) with binomial distribution was employed, considering the individual subjects as a random effect for all three conditions. All of the analyses were performed using the software R (v.3.2.3) with the packages 'Ime4', 'effects', 'plyr' and 'ggplot2.'

We used 'drinking' behavior as our dependent variable. For both the rewarded (est. $=3.68, z=$ $4.98, p<0.001)$ and the unrewarded-shape (est. $=1.04, z=1.98, p=0.048)$ conditions, the performance was above the chance level, whereas for the unrewarded-illusion condition, it was not (est. $=0.0007, z=$ $1.467, p=0.99)$.

To exclude the possibility that the learned tasks were extinguished after the animals were faced with unrewarded trials, we considered only the very first trial for each condition. For this analysis no random effect was included, given the fact that only one trial per condition for each animal is considered. The unrewarded-shape condition differed significantly from chance levels when limiting the analysis to the very first trial (est. $=1.3863, z=-2.15, p=0.031$ ), the unrewarded-illusion did not (est. $=-0.81, z=$ $1.35, p=0.18$; see Table 3, Figure 4).

Table 1

Number of Trials Performed, Successfully Recorded, Answered and Analyzed, and Relative Percentages, Divided for Conditions ( $R=$ Rewarded, US=Unrewarded-shape, UI=Unrewarded-illusion)

\begin{tabular}{lcccc}
\hline & R & US & UI & Total \\
\hline Trials performed & 552 & 138 & 138 & 828 \\
Trials recorded & 516 & 125 & 128 & 769 \\
Trials recorded (\% on performed) & $93.48 \%$ & $90.58 \%$ & $92.75 \%$ & $92.87 \%$ \\
Trials answered & 93 & 34 & 27 & 154 \\
Trials answered (\% on recorded) & $18.02 \%$ & $27.20 \%$ & $21.09 \%$ & $20.03 \%$ \\
Trials analyzed & 93 & 31 & 27 & 151
\end{tabular}

Note. The difference between percentages of answered trials among groups (18.02\%, 27.20\%, 20.03\%) is not statistically significant (proportional 3 sample test $-\chi^{2}(2)=5.3476, p=0.069$ ). 
Table 2

Number of Trials Performed, Answered and Correct for Each Spider for the Three Conditions

\begin{tabular}{|c|c|c|c|c|c|c|c|c|c|}
\hline \multicolumn{10}{|l|}{ Rewarded } \\
\hline Subject & S01 & S02 & S03 & S04 & S05 & S06 & S07 & S08 & S09 \\
\hline $\mathrm{N}$ & 22 & 23 & 34 & 35 & 45 & 45 & 43 & 44 & 32 \\
\hline Answered & 7 & 1 & 5 & 13 & 4 & 7 & 3 & 14 & 8 \\
\hline Correct & 7 & 1 & 5 & 13 & 4 & 7 & 3 & 14 & 8 \\
\hline Correct ( $\%$ on ans.) & $100 \%$ & $100 \%$ & $100 \%$ & $100 \%$ & $100 \%$ & $100 \%$ & $100 \%$ & $100 \%$ & $100 \%$ \\
\hline Subject & $\mathrm{S} 10$ & S11 & $\mathrm{S} 12$ & S13 & $\mathrm{S} 14$ & $\mathrm{~S} 15$ & S16 & S17 & S18 \\
\hline $\mathrm{N}$ & 11 & 33 & 33 & 10 & 34 & 12 & 23 & 24 & 12 \\
\hline Answered & 0 & 7 & 5 & 3 & 6 & 2 & 6 & 1 & 1 \\
\hline Correct & 0 & 6 & 4 & 3 & 6 & 2 & 5 & 1 & 1 \\
\hline Correct ( $\%$ on ans.) & - & $85.71 \%$ & $80.00 \%$ & $100 \%$ & $100 \%$ & $100 \%$ & $83.33 \%$ & $100 \%$ & $100 \%$ \\
\hline \multicolumn{10}{|l|}{ Unrewarded-shape } \\
\hline Subject & S01 & S02 & S03 & S04 & S05 & S06 & S07 & S08 & S09 \\
\hline $\mathrm{N}$ & 6 & 6 & 8 & 8 & 11 & 10 & 11 & 10 & 9 \\
\hline Answered & $2(3)$ & 1 & 4 & $1(2)$ & 1 & 2 & 2 & 1 & 3 \\
\hline Correct & 0 & 1 & 3 & 1 & 1 & 0 & 2 & 1 & 2 \\
\hline Correct (\% on ans.) & $0.00 \%$ & $100.00 \%$ & $75.00 \%$ & $100.00 \%$ & $100.00 \%$ & $0.00 \%$ & $100.00 \%$ & $100.00 \%$ & $66.67 \%$ \\
\hline Subject & S10 & S11 & $\mathrm{S} 12$ & S13 & S14 & $\mathrm{S} 15$ & S16 & S17 & $\mathrm{S} 18$ \\
\hline $\mathrm{N}$ & 1 & 9 & 8 & 2 & 8 & 3 & 6 & 6 & 3 \\
\hline Answered & 0 & 3 & 4 & $1(2)$ & 2 & 0 & 3 & 1 & 0 \\
\hline Correct & 0 & 3 & 3 & 1 & 0 & 0 & 3 & 1 & 0 \\
\hline Correct (\% on ans.) & - & $100.00 \%$ & $75.00 \%$ & $100.00 \%$ & $0.00 \%$ & - & $100 \%$ & $100 \%$ & - \\
\hline \multicolumn{10}{|c|}{ Unrewarded-illusion } \\
\hline Subject & S01 & S02 & S03 & S04 & S05 & S06 & S07 & S08 & S09 \\
\hline $\mathrm{N}$ & 6 & 5 & 10 & 8 & 10 & 11 & 12 & 12 & 8 \\
\hline Answered & 3 & 1 & 0 & 0 & 2 & 1 & 6 & 1 & 2 \\
\hline Correct & 3 & 1 & 0 & 0 & 1 & 0 & 1 & 0 & 2 \\
\hline Correct ( $\%$ on ans.) & $100.00 \%$ & $100.00 \%$ & - & - & $50.00 \%$ & $0.00 \%$ & $16.67 \%$ & $0.00 \%$ & $100.00 \%$ \\
\hline Subject & $\mathrm{S} 10$ & $\mathrm{~S} 11$ & $\mathrm{~S} 12$ & $\mathrm{~S} 13$ & S14 & $\mathrm{S} 15$ & $\mathrm{~S} 16$ & S17 & $\mathrm{S} 18$ \\
\hline $\mathrm{N}$ & 2 & 8 & 9 & 2 & 8 & 3 & 6 & 5 & 3 \\
\hline Answered & 0 & 2 & 2 & 0 & 1 & 2 & 2 & 0 & 2 \\
\hline Correct & 0 & 0 & 1 & 0 & 1 & 1 & 1 & 0 & 1 \\
\hline Correct (\% on ans.) & - & $0.00 \%$ & $50.00 \%$ & - & $100.00 \%$ & $50.00 \%$ & $50.00 \%$ & - & $50.00 \%$ \\
\hline
\end{tabular}

Note. The number in parentheses (Unrewarded-shape block, Answered row, spiders S01, S04, S13) represents the answered trials when including trials in which spiders drank both drops. 
Table 3

Binomial Tests Performed with Generalized Linear Model in " $R$ 3.3.2"

\begin{tabular}{|c|c|c|c|c|c|}
\hline$\underline{\mathrm{R} \text { - formula }}$ & Contrast & Estimate & SE & $Z$ & $p$ \\
\hline glmer(PERF COND+1 | SUBJ:COND) ) & $\mathbf{R} \leftrightarrow$ chance level & 3.6779 & 0.740 & 4.968 & $<0.001$ \\
\hline glmer(PERF COND+(1 | SUBJ:COND) ) & US $\leftrightarrow$ chance level & 1.043 & 0.528 & 1.976 & 0.048 \\
\hline glmer(PERF COND + (1 | SUBJ:COND) ) & $\mathrm{UI} \leftrightarrow$ chance level & 0.0007 & 0.498 & 0.001 & 0.999 \\
\hline $\operatorname{glm}($ PERF COND $)$ & $\mathbf{R} \leftrightarrow$ chance level & 2.773 & 1.031 & 2.69 & 0.007 \\
\hline $\operatorname{glm}($ PERF COND $)$ & US $\leftrightarrow$ chance level & $\mathbf{1 . 3 8 6 3}$ & 0.646 & 2.148 & 0.032 \\
\hline $\operatorname{glm}(\mathrm{PERF} \sim \mathrm{COND})$ & $\mathrm{UI} \leftrightarrow$ chance level & -0.8109 & 0.601 & -1.349 & 0.177 \\
\hline
\end{tabular}

Note. $\mathrm{R}$ - formula: formula used in $\mathrm{R}$ to fit the model, contrast: contrast to which the values are referred, estimate: coefficient value fitted on the binomial curve for given contrast, SE: standard error fitted on the binomial curve for given contrast, $\mathrm{PERF}=$ performance of spiders (correct or wrong), $\mathrm{COND}=$ experimental condition ( $\mathrm{R}=$ rewarded, US=unrewarded-shape, $\mathrm{UI}=$ unrewarded-illusion), SUBJ=subjects. Bold text represents contrast with $p<0.05$.

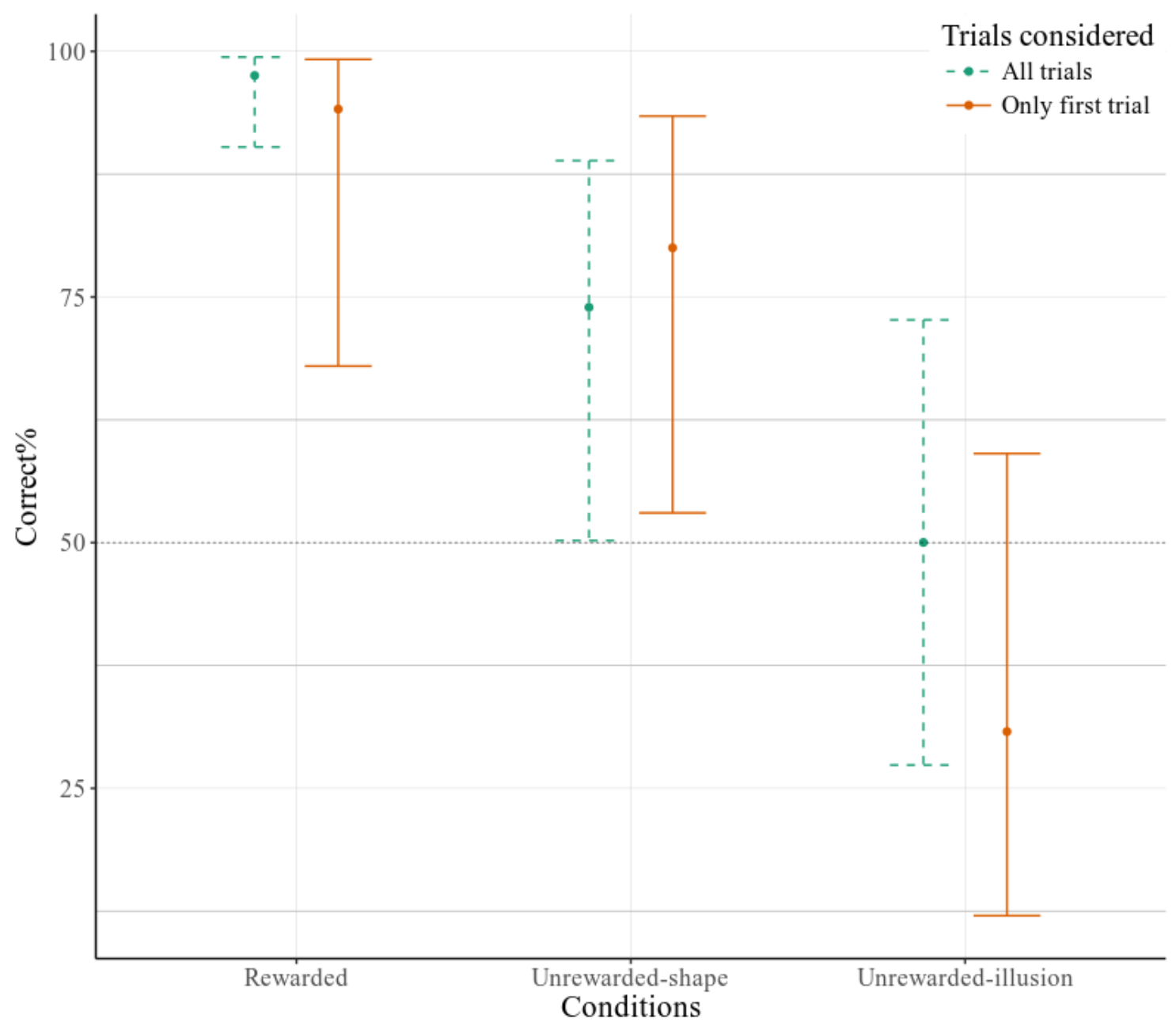

Figure 4. Correct performance (colored dots) of the spiders on the rewarded, unrewarded-shape and unrewarded-illusion trials, with confidence levels (95\%). The two models (considering all trials or only the first trial for each spider) are represented in different colors. Fifty-percent is the expected level of chance. For both models there is a significant difference from chance level for the rewarded condition (All trials p-value $<0.0001$, Only first trial p-value $=0.0071$ ) and for the unrewarded-shape condition (All trials $\mathrm{p}$-value $=0.048$, Only first trial $\mathrm{p}$-value $=0.031)$, but not for the unrewarded-illusion condition $($ All trials $\mathrm{p}$-value $=$ 0.99 , Only first trial p-value $=0.18$ ). 


\section{Discussion}

In the unrewarded-shape condition, we presented the spiders with the two training shapes but in the absence of any reward (sugar water or citric acid water). The performance above chance levels in this condition demonstrated that Phidippus regius was able to learn the association between an abstract, not biologically relevant stimulus and a reward. Spiders could not have just used ultra-violet reflection or chemical cues during learning. In fact, this information was absent during the unrewarded trials. This procedure seems to be a promising method for training spiders, a task that is known to be far from simple (Jakob \& Long, 2016).

We believe that success with this procedure depended on the modality of the presentation of the stimuli, which granted the spiders the possibility of freely inspecting the stimuli. In fact, both in the training and in the testing phase, the animals wandered in the experimental apparatus looking at both shapes and both drops multiple times prior to making their choices. They inspected the images from different distances and even touched the screens. This observation is coherent with the idea of a 'visual inspection' as Cross and Jackson (2016) proposed and as described in some Spartanae species prior to engaging in detour behaviors (Tarsitano, 2006; Tarsitano \& Andrew, 1999; Tarsitano \& Jackson, 1997). We think that this active and prolonged scanning behavior is fundamental for the process of learning novel visual stimuli, as well as in novel detours. In the study by Bednarski et al. (2012), described in the introduction, the spiders had no possibility of engaging in this visual inspection, given the fact that the stimuli were moving.

In the illusory condition, the spiders were presented with an occluded version of the previously rewarded stimulus versus a broken version of the same stimulus. The two stimuli were identical in their local features but differed in their global configurations (which, to the human eye, could be perceived only in the occluded stimulus). The spiders' performance at the chance level seems to indicate that the spiders were not using a global visual strategy, or the amodal completion mechanism, to identify the correct stimulus. Although preferential processing of local features has been described in various animal species (Fagot \& Parron, 2012), the mechanisms underlying such strategies are not fully understood. Spiders could provide a further and valuable model for their investigation. The use of a local strategy (i.e., basing the choice on the local features, which were identical in the two stimuli presented) is, however, not the only possible explanation. One alternative possibility is that the spiders had previously learned avoidance of the punished shape rather than association and approach of the positively rewarded one. When the spiders were presented with two versions of the positively rewarded shape in the illusory condition, neither stimulus could trigger avoidance (or the choice of the other stimulus) in spite of the use of a global visual strategy. However, further experiments are needed to test both of these hypotheses.

Unfortunately, we cannot draw any information from the learning trials in which sugar water and citric acid water were present (training phase and rewarded condition of the testing phase). Almost no spiders drank the citric acid drop because, we assume, they were able to easily recognize the taste as unpleasant beforehand with a simple touch. We considered using a different indicator of choice, such as the first drop approached, although we realized that approach does not constitute reliable information (see the 'Results' section). Detecting and scoring from the video recordings any simple touch of the drops was impossible. For this reason, we lacked a measure of the training progression. We are now implementing an automatic, computerized scoring method that is capable of detecting and recording even small contacts of the spiders with the drops. Such a method will optimize the objectivity and reliability of the behavioral measures (Döring \& Chittka, 2011). In fact, scoring is time consuming and relies heavily on the expertise of the scorers. In a few trials (10 out of the 769 recorded trials), we had to analyze the recordings multiple times and compare the opinions of all scorers because the animal's position or orientation made it hard to judge whether it was drinking or not.

In conclusion, we demonstrated that Phidippus regius can acquire a discrimination between artificial, abstract stimuli. The spiders, however, failed to discriminate the occluded version of the training shape versus its broken ('cut') version. Spiders seemingly relied on the local features rather than on the global configuration to identify the stimulus as they were shown (in previous studies) to do during the 
categorization of the prey versus non-prey. Further experiments are needed to control for the alternative interpretation. In the end, this experiment extends our knowledge about spiders' abilities in discrimination learning and describes a method that can be used for training the family of Salticidae.

\section{Acknowledgements}

We are grateful to students of our laboratory, Alba Liso, Joanna Krzyczkowska, Federica Dal Cin, for animal care, and to Andrea Badaile for the technical support.

Conflict of Interest: The authors declare that they have no conflict of interest.

\section{References}

Alem, S., Perry, C. J., Zhu, X., Loukola, O. J., Ingraham, T., Sevik, E., \& Chittka, L. (2016). Associative mechanisms allow for social learning and cultural transmission of string pulling in an insect. PLoS Biology, 14, e1002564. doi.org/10.1371/journal.pbio.1002564

Bednarski, J. V., Taylor, P., \& Jakob, E. M. (2012). Optical cues used in predation by jumping spiders, Phidippus audax (Araneae, Salticidae). Animal Behaviour, 84, 1221-1227. doi.org/10.1016/j.anbehav.2012.08.032

Cammaerts, M.-C., \& Cammaerts, R. (2015). Are ants (Hymenoptera, Formicidae) capable of self-recognition? Journal of Science, 5, 521-532.

Carducci, J., \& Jakob, E. M. (2000). Rearing environment affects behaviour of jumping spiders. Animal Behaviour, 59, 39-46. doi.org/10.1006/anbe.1999.1282

Cross, F. R., \& Jackson, R. R. (2014). Specialised use of working memory by Portia africana, a spider-eating salticid. Animal Cognition, 17, 435-444. doi.org/10.1007/s10071-013-0675-2

Cross, F. R., \& Jackson, R. R. (2016). The execution of planned detours by spider-eating predators. Journal of the Experimental Analysis of Behavior, 105, 194-210. doi.org/10.1002/jeab.189

Cross, F. R., \& Jackson, R. R. (2017). Representation of different exact numbers of prey by a spider-eating predator. Interface Focus, 7, 20160035. doi:10.1098/rsfs.2016.0035

Dacke, M., \& Srinivasan, M. V. (2008). Evidence for counting in insects. Animal Cognition, 11, 683-689. doi.org/10.1007/s10071-008-0159-y

De Voe, R. D. (1975). Ultraviolet and green receptors in principal eyes of jumping spiders. The Journal of General Physiology, 66, 193-207. doi.org/10.1085/jgp.66.2.193

Dolev, Y., \& Nelson, X. J. (2014). Innate pattern recognition and categorization in a jumping spider. PLoS ONE, 9 , e97819. doi.org/10.1371/journal.pone.0097819

Dolev, Y., \& Nelson, X. J. (2016). Biological relevance affects object recognition in jumping spiders. New Zealand Journal of Zoology, 43, 42-53. doi.org/10.1080/03014223.2015.1070183

Döring, T. F., \& Chittka, L. (2011). How human are insects and does it matter? Formosan Entomology, 31, 85-99. doi.org/10.6661/TESFE.2011008

Fagot, J., \& Parron, C. (2012). Visual cognition in baboons: Attention to global and local stimulus properties. In O. Lazareva, T. Shimizu, \& E. A. Wasserman (Eds.), How animals see the world: Comparative behavior, biology, and evolution of vision (pp. 370-385). Oxford, UK: Oxford University Press.

Friard, O., \& Gamba, M. (2016). BORIS: A free, versatile open-source event-logging software for video/audio coding and live observations. Methods in Ecology and Evolution, 7, 1325-1330. doi.org/10.1111/2041210X.12584

Hammer, M., \& Menzel, R. (1995). Learning and memory in the honeybee. The Journal of Neuroscience, 15, 16171630. doi.org/10.1016/j.cub.2005.09.015

Harland, D. P., Li, D., \& Jackson, R. R. (2012). How jumping spiders see the world. In O. Lazareva, T. Shimizu, \& E. A. Wasserman (Eds.), How animals see the world: Comparative behavior, biology, and evolution of vision (pp. 132-163). Oxford, UK: Oxford University Press.

Herberstein, M. E. (2011). Spider behaviour: Flexibility and versatility (1st ed). Cambridge: Cambridge University Press.

Jakob, E. M., \& Long, S. (2016). How (not) to train your spider: Successful and unsuccessful methods for studying learning. New Zealand Journal of Zoology, 43, 112-126. doi.org/10.1080/03014223.2015.1127263

Jakob, E. M., Skow, C. D., Haberman, M. P., \& Plourde, A. (2007). Jumping spiders associate food with color cues 
in a T-maze. Journal of Arachnology, 35, 487-492. doi.org/10.1636/JOA-ST06-61.1

Kanizsa, G. (1979). Organization in vision: Essays on Gestalt perception. New York: Praeger.

Kanizsa, G., Renzi, P., Conte, S., Compostela, C., \& Guerani, L. (1993). Amodal completion in mouse vision. Perception, 22, 713-721. doi.org/10.1068/p220713

Land, M. F. (1969). Movements of the retinae of jumping spiders (Salticidae: Dendryphantinae) in response to visual stimuli. Journal of Experimental Biology, 51, 471-493.

Liedtke, J., \& Schneider, J. M. (2014). Association and reversal learning abilities in a jumping spider. Behavioural Processes, 103, 192-198. doi.org/10.1016/j.beproc.2013.12.015

Menda, G., Shamble, P. S., Nitzany, E. I., Golden, J. R., \& Hoy, R. R. (2014). Visual perception in the brain of a jumping spider. Current Biology, 24, 2580-2585. doi.org/10.1016/j.cub.2014.09.029

Menzel, R., Greggers, U., Smith, A., Berger, S., Brandt, R., Brunke, S., ...Watzl, S. (2005). Honey bees navigate according to a map-like spatial memory. Proceedings of the National Academy of Sciences of the United States of America, 102, 3040-3045. doi.org/10.1073/pnas.0408550102

Michotte, A., Thines, G., \& Crabbe, G. (1964). Les complements amodaux des structures perceptives [Amodal complements of perceptual structures]. Louvain, Belgium: Publications Universitaires de Louvain.

Nelson, X. J., \& Jackson, R. R. (2012). The role of numerical competence in a specialized predatory strategy of an araneophagic spider. Animal Cognition, 15, 699-710. doi.org/10.1007/s10071-012-0498-6

Peckmezian, T., \& Taylor, P. W. (2015a). Electric shock for aversion training of jumping spiders: Towards an arachnid model of avoidance learning. Behavioural Processes, 113, 99-104.

Peckmezian, T., \& Taylor, P. W. (2015b). A virtual reality paradigm for the study of visually mediated behaviour and cognition in spiders. Animal Behaviour, 107, 87-95. doi.org/10.1016/j.anbehav.2015.06.018 doi.org/10.1016/j.beproc.2015.01.015

Regolin, L., \& Vallortigara, G. (1995). Perception of partly occluded objects by young chicks. Perception \& Psychophysics, 57, 971-976. doi.org/10.3758/BF03205456

Sato, A., Kanazawa, S., \& Fujita, K. (1997). Perception of object unity in a chimpanzee (Pan troglodytes). Japanese Psychological Research, 39, 191-199. doi.org/10.1111/1468-5884.00053

Sovrano, V. A., \& Bisazza, A. (2008). Recognition of partly occluded objects by fish. Animal Cognition, 11, 161166. doi.org/10.1007/s10071-007-0100-9

Tarsitano, M. (2006). Route selection by a jumping spider (Portia labiata) during the locomotory phase of a detour. Animal Behaviour, 72, 1437-1442. doi.org/10.1016/j.anbehav.2006.05.007

Tarsitano, M., \& Andrew, R. (1999). Scanning and route selection in the jumping spider Portia labiata. Animal Behaviour, 58, 255-265. doi.org/10.1006/anbe.1999.1138

Tarsitano, M., \& Jackson, R. R. (1997). Araneophagic jumping spiders discriminate between detour routes that do and do not lead to prey. Animal Behaviour, 53, 257-266. doi.org/10.1006/anbe.1996.0372

van Hateren, J. H., Srinivasan, M. V., \& Wait, P. B. (1990). Pattern recognition in bees: Orientation discrimination. Journal of Comparative Physiology, 167, 649-654. doi.org/10.1007/BF00192658

Wehner, R. (2003). Desert ant navigation: How miniature brains solve complex tasks. Journal of Comparative Physiology, 189, 579-588. doi.org/10.1007/s00359-003-0431-1

Zylinski, S., Darmaillacq, A.-S., \& Shashar, N. (2012). Visual interpolation for contour completion by the European cuttlefish (Sepia officinalis) and its use in dynamic camouflage. Proceedings of the Royal Society B: Biological Sciences, 279, 2386-2390. doi.org/10.1098/rspb.2012.0026 